\title{
Tratamiento integral del Trastorno Límite de Personalidad.
}

\author{
Comprehensive treatment of Borderline Personality Disorder.
}

\begin{abstract}
Mª Teresa García López ${ }^{\mathrm{a}}$ M M $^{\mathrm{a}}$ Fe Martín Pérez ${ }^{\mathrm{b}}$, Raúl Otín Llop
${ }^{a}$ Médico Residente Psiquiatría. CSMIJ-Olot. XSM-Parc Hospitalari Martí i Julià. ${ }^{b}$ Médico Adjunto Psiquiatría. Jefe de Sector CSMA-La Selva Interior. 'Médico Adjunto Psiquiatría. Jefe de de Hospitalización. XSM-Parc Hospitalari Martí i Julià.
\end{abstract}

Correspondencia: Ma Teresa García López, (maitegl1975@hotmail.com)

\begin{abstract}
RESUMEN: Los Trastornos de Personalidad (TP) en general y el Trastorno Límite de Personalidad (TLP) en particular, son de difícil abordaje ya que los trastornos de personalidad no están considerados una enfermedad mental como tal al tratarse de patología de eje II; se necesita un tratamiento psicofarmacológico y psicoterapéutico ("abordaje integral") en muchas ocasiones de forma crónica, evidenciándose una falta de recursos y estudios sobre el mismo a largo plazo. También hay que señalar las dificultades en la práctica clínica de manejo del TLP, en particular por las propias características de éste (impulsividad, inestabilidad,...) que en muchas ocasiones provocan fenómenos de contratransferencia difíciles de controlar por el profesional. Este artículo pretende ser una revisión del TLP y su tratamiento integral en los diferentes niveles asistenciales pero centrado en hospitales de día, ya que en general es en estos dispositivos donde se tratan los casos más graves y donde más implantados están los programas específicos (si no para los TLP en particular sí para los TP en general). Finalmente en los Centros de Salud Mental, se están realizando ya grupos específicos para TP como parte de su tratamiento integral a este nivel.
\end{abstract}

PALABRAS CLAVE: Trastorno límite de personalidad, borderline, trastorno de personalidad, tratamiento integral, abordaje integral, programas para trastornos de personalidad.
ABSTRACT: In general Personality Disorders (PD) and Borderline Personality (BP) especially, don't have an easy boarding since: personality disorders are not considered a mental illness as such; a psychopharmacologic and psychotherapeutic ("integral boarding") treatment is needed, frequently in chronic form; and by the other side, is demostrated a lack of resources and studies at longterm. Also, there are difficulties in clinical practice for the managing patients with BP, especially for their own characteristics (impulsiveness, instability, ...) that, in many occasions, provoke countertransfer phenomena's difficult to control for the professional. This article tries to be a review of the BP and its integral treatment in the different levels welfare but centred on day hospitals. Generally, is in these devices where the most serious cases are treated and where the specific programs are wellestablished (but for the BP especially yes for the $\mathrm{PD}$ in general). Finally to mention that in ambulatory level, in Mental Health Centers, specific groups for PD are realized as a part of its integral treatment in this level.

KEY WORDS: Borderline Personality Disorder, comprehensive treatment programs for personality disorders.

\section{Personalidad}

La palabra persona deriva del griego prosopon (cara, rostro, aspecto) que en el etrusco pasó como phersu, siendo la definición inicial de "persona" la máscara de teatro con la que los actores imitaban la fisonomía de quien representaban, adquiriendo posteriormente el significado del papel representado y finalmente definiendo aquél al que representaban.

El término personalidad aparece el contexto del florecimiento del cristianismo, al concebir a Dios como 3 personas (padre, hijo y espíritu santo). En otras

Recibido: 15/10/2008; aceptado con modificaciones: 29/01/2010

Rev. Asoc. Esp. Neuropsiq., 2010; 30 (106), 263-278. 
palabras, la personalidad es la esencia que se encuentra en la apariencia. La Real Academia de la Lengua (RAE) la define como la diferencia individual que constituye a cada persona y la distingue de otra.

\section{El trastorno límite de personalidad:}

Para empezar a hablar de los TP se debe primero definir qué es la personalidad. La personalidad es un constructo multifactorial dinámico con un componente biológico y un componente ambiental interrelacionados. Es decir, la personalidad consta de una parte heredada o genética (el temperamento) y otra social y ambiental (el carácter). Posee características tan opuestas como ser individual (idiosincrásica a la persona) y social (aprende del entorno y el entorno de ella), estable durante la existencia del individuo pero a la vez facilitando la adaptación de éste al entorno.

Es lógico suponer que la alteración de cualquiera de estos componentes puede dar lugar a un TP; y en el caso del TLP la vulnerabilidad biológica, las experiencias psicológicas traumáticas y un contexto social inadecuado pueden dar lugar a dicho trastorno. Pero esto no siempre es así y aunque se den factores considerados necesarios para desarrollar un TP también existen factores protectores. Así, si un individuo posee vulnerabilidad biológica pero su entorno familiar es adecuado y no presenta experiencias traumáticas psicológicas puede no llegar a presentar un TP.

El término diagnóstico de límite (borderline) surge en el marco del psicoanálisis. El primer autor en utilizar este término fue Stern en 1938, pero Pinel en 1801 fue el primero en hacer constatar por escrito sintomatología compatible con TLP (denominándola "manie sans délire"). Las orientaciones posteriores al psicoanálisis también han realizado aportaciones en relación a esta patología. Dentro de la orientación biológica destacan Akiskal, Siever y Davis y Widiger entre otros y dentro de la orientación biopsicosocial Cloninger, Millon y Linehan. Cada orientación engloba el TLP según su teoría. Así, desde una perspectiva psicoanalítica se encontraría entre la neurosis y la psicosis, mientras que desde una visión biológica se enmarcaría dentro del espectro de los trastornos afectivos. En otras orientaciones el TLP se considera una mezcla grave, pero estable, de diferentes subtipos de personalidad. En la actualidad el concepto de "límite" abarca clínica de casi cualquier patología psiquiátrica incluyendo la esfera psicótica, la esfera afectiva, desorganización grave de la personalidad, la esfera de los trastornos impulsivos y finalmente se ha relacionado con el estrés postraumático.

La prevalencia en la población general del TLP se sitúa entre un 1-2\%, mientras que en los pacientes ambulatorios de CSM y en los hospitalizados se sitúa entre el 11-20\% y el 18-32\% respectivamente. El TLP es tres veces más frecuente en mujeres, con una mayor prevalencia cuando se asocian un entorno urbano y un 
ORIGINALES Y REVISIONES

bajo nivel socioeconómico. La edad de diagnóstico del TLP se sitúa entre los 1932 años, siendo menos frecuente su diagnóstico a mayor edad pero en estos casos, normalmente, implica mayor gravedad del trastorno. En contextos comunitarios se ha asociado a más experiencias traumáticas, a la existencia de abusos físicos y/o sexuales infantiles, a mayor frecuencia de relaciones homosexuales y dudas sobre la identidad sexual, a antecedentes personales y/o familiares de ingresos psiquiátricos ( $\sin$ haber encontrado una mayor asociación con la esquizofrenia que en la población general).No se ha confirmado a asociación significativa con los trastornos afectivos con un mayor número de tentativas autolíticas ni con una mayor prevalencia de abuso de sustancias.

En los últimos años existe un incremento de atención especializada para los TP, especialmente en el caso de los TLP, que ha provocado: un aumento de los recursos destinados (aunque falta por determinar si éstos son suficientes o demasiados) y que se esté replanteando su clasificación (diagnóstico categorial o dimensional). En el caso del TLP se está valorando su inclusión como patología de eje I en el DSM-V.

Como se ha comentado anteriormente lo único que decirse con certeza de la etiología de los TP es que no está clara. Desde los modelos más biologicistas en la búsqueda de marcadores biológicos que faciliten el estudio y tratamiento de este trastorno, pasando por los modelos biopsicosociales (Eysenk, Cloninger, SieverDavis,etc...) que postulan diferentes dimensiones de la personalidad y sus interacciones como base de los diferentes TP y sus diferentes grados de gravedad, hasta los modelos psicológicos/psicosociales que englobarían los modelos psicoanalíticos (Kernberg, Fonagy y Steele, etc...), los modelos interpersonales y los modelos cognitivo-conductuales (Beck y Freeman, Young, Linehan, etc...) que hipotetizan el origen de los TP en los conflictos madre-hijo, en interacciones inadecuadas con el entorno y en el aprendizaje de cogniciones y conductas patológicas que deben aprender a reconocer para poder ser tratados y ser resueltos; todos, de alguna manera, intentan explicar la causa de los TP con la intención de facilitar su estudio y tratamiento.

Cabe señalar, que cuando se diagnostica un TP (incluido el TLP) debe haberse realizado un seguimiento longitudinal del individuo, ya que muchos con determinados rasgos caracteriales (sin ser patológicos) en determinados momentos y en situaciones estresantes pueden presentar un incremento de estos rasgos, incluso llegando a ser desadaptativos sin que esto implique un diagnóstico deTP. El diagnóstico de TP implica además de la presencia a lo largo del tiempo de rasgos patológicos, una disfunción global (en cualquier ámbito de la vida diaria) que causa malestar al paciente. Además, el hecho de estar diagnosticado de TP no implica la existencia constante de esta disfuncionalidad. De hecho, la gran mayoría de pacientes permanecen en un frágil equilibrio y cualquier estresor puede provocar que en- 
tren en "crisis", apareciendo inestabilidad, intensificándose los rasgos caracteriales desadaptativos, incrementándose la disfuncionalidad global y apareciendo un gran malestar.

La palabra clave para definir el TLP es la inestabilidad, lo que en un primer momento resulta paradójico si se tiene en cuenta que una de las condiciones básicas para diagnosticar un TP es que cumpla unos criterios determinados de forma estable. Esto, que en el caso del TLP podría parecer contradictorio, puede entenderse si asumimos que lo "estable" en el TLP es precisamente esa inestabilidad. En el TLP dicha inestabilidad se aplica a cualquier aspecto de la vida del paciente: afectiva (afecto intenso, sentimientos crónicos de vacío, anhedonia, etc...), interpersonal (superficialidad de las relaciones, dependencia, manipulación, etc...), conductual (impulsividad con autolesiones, tentativas autolíticas, abuso de tóxicos, promiscuidad, etc...) y cognitiva (la menos frecuente pudiendo presentar episodios psicóticos breves). Como puede suponerse no todos TLP presentan toda la clínica descrita, sino que predominan unos rasgos sobre otros y dependiendo del momento vital, cualquiera de ellos pueden presentar mayor o menor intensidad.

Otro punto importante del TLP es la frecuente existencia de comorbilidad con otros trastornos del eje I y del mismo eje II. Dentro del eje I la comorbilidad es mayor con trastornos de la esfera afectiva (sobre todo la Depresión Mayor) y trastornos de ansiedad (destacando el Trastorno por Estrés Post-Traumático sobre los demás) con una frecuencia de alrededor del $90 \%$ ambos, seguidos del trastorno por abuso de sustancias (en un 50\% de los casos alcohol) y de trastornos alimentarios (sobre todo el trastorno de conducta alimentaria no especificado) con una frecuencia de $60 \%$ y 50\% aproximadamente cada uno. Por otro lado, el TLP puede coexistir con otro TP dentro del eje II, siendo los más frecuentes los de Clúster C (sobre todo el trastorno de personalidad dependiente) en más de un $70 \%$ de los casos. Por géneros, la comorbilidad dentro del eje I y del eje II es diferente. Siendo en los hombres más frecuente el trastorno por abuso de sustancias (sobretodo alcohol) y en mujeres el trastorno de conducta alimentaria dentro del eje I. Dentro del eje II los trastornos de personalidad de clúster A o B son más frecuentes en varones con TLP.

No se puede acabar de hablar de la clínica del paciente con TLP sin hacer referencia al suicidio, a las tentativas y/o gestos autolíticos y a los actos parasuicidas que, se englobarían, en general, como uno de los síntomas que más se asocian, al TLP. Y que de hecho, forma parte de los criterios diagnósticos de la enfermedad. La mayoría de los pacientes con TLP realizan tentativas autolíticas (entre 3-4 por paciente). La tasa de suicidio consumado en estos pacientes es elevada, 8-10\% de los pacientes (0.5-2\% en la población general), mientras que los gestos autolíticos están presentes en un 75\% (3-5\% en la población general). La presencia de gestos autolíticos se relaciona con: inestabilidad emocional crónica, así como con la pre- 
ORIGINALES Y REVISIONES

sencia de incremento de la irritabilidad, las alteraciones conductuales (destacando conductas de riesgo) y falta de soporte sociofamiliar.

Si por un lado la clínica del TLP puede asociarse, e incluso, confundirse con cualquier otra patología psiquiátrica, su diagnóstico no es más fácil. Actualmente el diagnóstico de TLP es un diagnóstico categorial (deben cumplirse un número determinado de ítems un tiempo determinado para considerar la posibilidad de diagnosticar un TLP), además, para complicar aún más su diagnóstico el clínico dispone de dos clasificaciones posibles: el DSM-IV (en general más usado en Norte América) y el CIE-10 (más usado en Europa). El diagnóstico categorial, si embargo, posee algunos inconvenientes: no tiene en cuenta el grado de sintomatología ni la gravedad del trastorno, no tiene en cuenta la posibilidad de comorbilidad con otros trastornos de eje I o II y en muchas ocasiones se observa un solapamiento de diferentes categorías. Por otra lado, algunos autores trabajan con otro tipo de clasificación: la dimensional (modelo de los Cinco grandes factores, modelo de Livesly, modelo de Cloninger y modelo biopsicosocial que en diferencia al anterior presentan las ventajas de las que carecen los modelos categoriales lo que, en teoría, les permitiría una mejor aplicación en la clínica y en la investigación facilitando un tratamiento psicofarmacológico y psicoterapéutico más eficaz. Finalmente, si el TLP presenta clínica de casi cualquier patología psiquiátrica, es lógico suponer que su diagnóstico diferencial es difícil (esquizofrenia, trastornos afectivos, TEPT, trastornos por abuso/dependencia de alcohol y/u otros tóxicos, trastornos somatomorfos, parafilias, trastornos del control de impulsos y otros trastornos de personalidad). Como vemos, todas estas dificultades son las que priman que el diagnóstico de los TP, y del TLP en particular, deba realizarse de forma longitudinal.

En general el pronóstico del paciente con TLP a corto/medio plazo va muy asociado a la correcta realización de un tratamiento psicoterapéutico y/o psicofarmacológico (50\% mejoran de la clínica) y a la abstinencia de sustancias (apreciándose un mayor porcentaje de mejoría, remitiendo de forma destacada la impulsividad con poca remisión de la sintomatología más afectiva y mejorando las relaciones interpersonales. A largo plazo, con un adecuado tratamiento es cuando se hace evidente la buena evolución del paciente disminuyendo la intensidad de la sintomatología, con una importante disminución del porcentaje de pacientes con diagnóstico de TLP que siguen cumpliendo sus criterios (menos de un 25\%) y un funcionamiento más adaptado (pero mayor fragilidad que los individuos sin TLP). Como factor predictor de buen pronóstico a largo plazo podemos utilizar la puntación en las escalas de impulsividad (DIB-R) y de funcionamiento global (GAF), y así a menor puntuación DIB-R y mayor GAF mejor pronóstico del paciente a largo plazo.

En relación al pronóstico, si tenemos en cuenta que los pacientes con TLP tienen tendencia a presentar con mayor frecuencia unos síntomas que otros, pode- 
mos formar dos grupos de síntomas. Un primer grupo donde destacaría la tendencia a las tentativas autolíticas, las autolesiones, los pensamientos casi-psicóticos, las regresiones con el tratamiento y problemática intensa de contratransferencia y que serían los que presentaría más manifestaciones agudas del TLP, más número de crisis y más necesidad de hospitalizaciones pero que por otro lado sería el de mayor facilidad diagnóstica. Y un segundo grupo donde resaltarían los sentimientos crónicos de vacío o rabia, el miedo a la soledad o al abandono y la suspicacia, que podemos imaginar que serán de más difícil diagnóstico y por lo tanto su resolución y su curso serán más lentos. Como vemos, sea cual sea, el grupo al que pueda pertenecer un paciente con TLP el pronóstico a largo plazo conlleva una remisión (como mínimo parcial de la sintomatología). $(1,2,3)$

\section{Intervenciones terapéuticas:}

El tratamiento del paciente con TLP puede abordarse de diversas maneras pero en general los pacientes con sintomatología poco intensa y/o un adecuado nivel funcional pueden beneficiarse de un tratamiento ambulatorio centrado en psicoterapia individual, precisando o no tratamiento psicofarmacológico. Mientras que los pacientes con intensa sintomatología y/o un pobre nivel funcional precisan de un tratamiento multidisciplinar más intensivo centrado en psicoterapia individual, familiar y grupal junto con tratamiento psicofarmacológico coadyuvante que se administra en unidades más especializadas (Hospitales de Día, Unidades de Hospitalización). En ocasiones, dada las dificultades de contención y la inestabilidad del paciente es preciso su ingreso. Resumiendo, se disponen de diferentes niveles asistenciales según la gravedad del paciente: Centro de Salud Mental (bajo riesgo autolítico, buen funcionamiento y soporte adecuado) y Hospital de Día (alto riesgo autolítico y falta de soporte); y en cada uno de los diferentes niveles se aplican diferentes programas terapéuticos: en CSM psicoterapia individual y/o grupal y en Hospital de Día psicofármacos, psicoterapia individual y/o grupal, grupos psicoeducativos, terapia de familia, etc. Así pues, el abordaje del TLP requiere de un plan de tratamiento integral. Desde una visión más sencilla, podemos entender el tratamiento integral como el tratamiento con psicoterapia y/o fármacos aplicado a un paciente con TLP. Avanzando un poco más en la complejidad del tratamiento específico del paciente con TLP se puede determinar que los programas específicos (en CSM o en Hospital de Día p. Ej. constituyen un tratamiento integral. En otro grado mayor de complejidad el tratamiento integral del TLP incluye la implicación y la coordinación entre los diferentes dispositivos de la red local de salud mental (CSM, Hospital de Día, Unidades de Hospitalización, Centros de Adicción a las Sustancias, etc...). En definitiva, el tratamiento integral del TLP incluye todo lo 
ORIGINALES Y REVISIONES

anterior. Una vez planteado el tipo de tratamiento idóneo para el TLP, cabe preguntarse por la evaluación de sus resultados. La falta de referencias en este sentido puede guardar relación con la gran complejidad de diseñar estudios que abarquen de forma global todos los aspectos implicados en el tratamiento integral del TLP. Sí que se han publicado estudios y artículos en relación con algunos de los aspectos implicados en el tratamiento del TLP (fármacos, programas y psicoterapias, siendo estas últimos los más frecuentes) y posteriormente se hará referencia a algunos de ellos, enfatizando los que se aplican en Hospital de Día donde se realizan tratamientos para los TP y específicamente, en algunos de ellos, para los TLP. (4)

Debemos mencionar, sin ser la parte más importante del tratamiento del TLP, las características generales del tratamiento psicofarmacológico. No existe ningún trabajo que haya demostrado ninguna especificidad o eficacia por ningún tratamiento farmacológico específico y esto podría estar en relación con dificultades al diseñar los estudios. En primer lugar por la gran variabilidad de criterios diagnósticos y la heterogeneidad clínica, en segundo lugar por la alta frecuencia de comorbilidad de eje I y/o II con el TLP y finalmente por la alta tasa de abandonos y de incumplimiento terapéutico asociada a la alta inestabilidad del paciente con TLP. Debido a todo lo anterior, el uso de un determinado fármaco no puede ayudar a definir el subtipo clínico del paciente, por lo que los objetivos del tratamiento farmacológico serían tratar el síntoma estado y la vulnerabilidad del rasgo. Pueden utilizarse desde ISRS, antipsicóticos, Litio y eutimizantes a otros más recientes como la Naltrexona y los ácidos grasos Omega-3 que ayudarían al control de la impulsividad y finalmente en casos de comorbilidad con trastorno depresivo mayor grave incluso TEC (terapia electroconvulsiva). Los fármacos que deben usarse con mucho cuidado son las benzodiacepinas (BZD) ya que con mayor frecuencia que en la población general, se dan reacciones paradójicas asociadas presentando inquietud y agitación en vez de efecto ansiolítico. $(5,6)$

No cabe duda, que la parte más importante del tratamiento del TLP es la psicoterapia. En el TLP se trabaja desde dos orientaciones psicoterapéuticas diferentes: la Psicodinámica (como ejemplos de grupos de esta orientación tenemos la Mentalización de Bateman y Fonagy y la Terapia Focalizada en la Transferencia de Kernberg y Clarkin) y la Cognitivo-conductual (como la Terapia Cognitiva para los TP de Beck y Freeman, la Terapia de Esquemas de Young, la Terapia Dialéctico Conductual de Linehan y la Terapia Icónica de Soledad Santiago) (7). Pueden encontrarse en la literatura multitud de trabajos que evalúan diferentes psicoterapias para el tratamiento del TP y del TLP en los que dadas las dificultades para comparar una determinada psicoterapia con un placebo (el no intervenir podría considerarse una intervención en sí y no se ha consensuado una psicoterapia estándar) se opta por comparar una determinada psicoterapia con otra y evaluar si significativamente una es más eficaz que la otra $(8,9,10)$. Como ejemplo de lo anterior de Kernberg y 
Clarkin en 2007 (11) en el que publicaban los resultados de un ensayo clínico randomizado realizado por ellos en el que se comparaban tres psicoterapias diferentes a nivel ambulatorio para TLP: la dialéctico conductual (TDC), la psicodinámica de soporte y la centrada en la transferencia (TFP) y que concluía que: aunque las tres intervenciones mejoraban la ansiedad, la depresión y el funcionamiento global, la TFP mejoraban el funcionamiento en más variables que las otras dos (destacando la disminución de conducta suicida, la ira y la impulsividad, la irritabilidad y la heteroagresividad) sin concretar qué mecanismos permitían esta mejoría. Como caso anecdótico, y por eso se cita, Paris en 2007(12) publicó un trabajo en el que describe dos casos ambulatorios en los que se realizó "psicoterapia intermitente", es decir se atendieron de forma aislada a pacientes en fase de crisis sin poderse realizar seguimiento posteriormente (lo que en la práctica clínica es bastante habitual). Paris, postula que dado que en la mayoría de los pacientes con TP las intervenciones a nivel ambulatorio en la práctica suelen ser de este tipo sin que posteriormente haya un empeoramiento del funcionamiento del paciente quizás este tipo de intervenciones podrían aplicarse a casos de TP no graves en los que la sintomatología remite con el tiempo (como por ejemplo el TLP) permitiendo un tratamiento breve $\mathrm{y}$ facilitando el retorno del paciente si fuese preciso.

\section{Programas integrales:}

Como se dijo al inicio del artículo quizás donde más puede aplicarse el término de tratamiento integral es en los Hospitales de Día, ya que es en este dispositivo donde se derivan los casos de TP graves que no mejoran o son incapaces de vincularse al tratamiento ambulatorio y que precisan de programas especiales para $\mathrm{TP}$ por lo que se debe realizar un abordaje integral. Normalmente se trata de pacientes con TP que presentan un mal funcionamiento sociofamiliar, falta de actividad laboral, rasgos de personalidad de Cluster B y/o una alta comorbilidad de trastorno por abuso de sustancias y trastornos psicóticos. Para entender qué es un abordaje integral y cómo se aplica creemos necesario poner algunos ejemplos de programas para dichos trastornos en Hospitales de Día. En el Reino Unido destaca el Hospital de Halliwick (A) donde el programa para los TLP incluye grupos de psicoterapia con mayor o menor número de pacientes según las necesidades terapéuticas, grupo de terapia expresiva (psicodrama), psicoterapia individual, tratamiento psicofarmacológico sintomático y supervisión de equipo.

En España se hallan de forma más o menos detallada algunos programas de hospital de día para TLP. En general en cada Hospital de día el programa de tratamiento para TP/TLP que se realiza constan de psicoterapia individual (generalmente de orientación más psicoanalítica o ecléctica), psicoterapia de grupo (ge- 
ORIGINALES Y REVISIONES

neralmente de orientación cognitivo-conductual), tratamiento psicofarmacológico sintomático, psicoterapia de familia y/o pareja, terapia ocupacional, supervisiones, etc... presentando cada uno sus peculiaridades. Así, en el CAEM (Consorcio Asistencial Emili Mira de Santa Coloma de Gramenet) (B) destaca la presencia de grupos relacionales (de orientación psicoanalítica que utiliza la mentalización) y grupos de habilidades (TDC de Linehan). En la Unidad de Trastornos de Personalidad de Cantabria(C) destaca la utilización de un programa en el que el tratamiento psicoterapéutico es exclusivo siguiendo una orientación psicoanalítica (Cognitivo analítica) denominado Psicoterapia Multicomponente de los Trastornos de Personalidad (C. Mirapeix). En el Centro Asistencial de San Juan de Dios en Málaga (D) como aportación propia se terapia icónica, un tipo de terapia cognitivo analítica creada por Soledad Santiago y que consta de técnicas cognitivas (reestructuración), técnicas conductuales (entrenamiento) y el uso de imágenes gráficas (Iconos) con el objetivo de facilitar la compresión de las 2 técnicas anteriores facilitando la memorización de éstas y la anticipación al impulso. En el Centro de Salud Mental de Arganda (E) se realiza un Programa Integrado para TP que consta de diferentes fases y donde destacan los grupos psicoeducativos de familias y el programa rehabilitador sociolaboral que realizan; además dependiendo de la evolución del paciente al alta puede realizarse una fase de seguimiento en que se mantiene contacto telefónico con el paciente, se realizan visitas a domicilio y se mantienen las coordinaciones con el CSM. El último programa para TP realizado en Hospital de Día que se cita corresponde al del Hospital de Día de Salt (Parc Hospitalari Martí i Julià) en Girona (F). No se trata de un programa para TLP como tal, pero sí para el TP en general. Se realizan actividades comunes como el grupo de buenos días, prensa y actualidad, creatividad literaria, Cine forum, debate, asamblea y taller de música. Cada una de estas actividades tiene una finalidad específica y se trabajan aspectos diferentes del trastorno del paciente (por ejemplo, conectar con sus emociones). El programa para TP consta de actividades individuales (que dependerán de las necesidades especificas del paciente), grupo de TP de orientación TDC, psicoterapia individual, grupo de relajación, grupo de autocontrol, expresión creativa, técnicas de autoconocimiento, ABVD (actividades básicas de la vida diaria), coordinaciones semanales con el sector de referencia y supervisiones mensuales.

Como resumen de los diferentes programas en Hospital de Día remarcaremos que en todos los citados se realiza psicoterapia individual y de grupo (independientemente de la orientación), tratamiento psicofarmacológico sintomático, trabajo de las habilidades sociales, trabajo en terapia ocupacional, y finalmente supervisión de los casos.

Por otro lado, en los últimos años han comenzado a formarse unidades de ingreso específicas para los TP con diferentes reacciones por parte de los profesionales de salud mental: aspectos controvertidos, como su consideración de enfermos mentales con el riesgo de desresponsabilización que ello comporta o incluso 
la propia eficacia de los mismos son aspectos que aún están siendo debatidos por la comunidad científica. Por otro lado, se ha sugerido que las unidades de hospitalización específicas para trastornos graves de personalidad (TGP) son eficaces, disminuyendo el número de reingresos en unidades de agudos, el número de visitas a urgencias y el "hospitalismo" de estos pacientes. Los ingresos en estas unidades son siempre programados, voluntarios (se debe ser mayor de edad y firmar un contrato terapéutico), de una duración de meses y con unos objetivos terapéuticos específicos (a diferencia de las unidades de hospitalización de agudos donde los ingresos de los pacientes con TP suelen ser vía urgencias y breves, con la finalidad de estabilizar al paciente). Dichos objetivos incluyen una intervención biopsicosocial (holística), destacando el trabajo a nivel individual, familiar, social y con la red de salud mental de referencia. En España el referente es la Unidad para TGP de Zaragoza (G) en la que, además de las condiciones generales citadas para ingresar, se precisa de una ausencia de dependencia de tóxicos, que el paciente no esté diagnosticado o no presente comorbilidad con el trastorno antisocial de la personalidad, que no existan causas o cargas legales pendientes y que el núcleo familiar o de convivencia se implique en el proceso terapéutico. De forma específica se trabajan objetivos como: establecer una buena alianza terapéutica con el paciente, confirmar el diagnóstico, tratar las conductas autodestructivas, trabajar la adherencia al tratamiento psicoterapéutico y/o farmacológico, trabajar la conciencia de trastorno, psicoeducación sobre el trastorno y finalmente se enseña al paciente a identificar y controlar los síntomas de forma que se pueda de obtener y mantener una estabilidad clínica.

Para lograr los objetivos anteriores se realizan una intervenciones específicas: se inicia tratamiento psicofarmacológico si precisa, se realizan psicoterapia individual y grupal así como grupos psicoeducativos, también se realiza intervención familiar, intervención de servicios sociales (si es necesario), terapia ocupacional y finalmente durante todo el proceso se trabaja la responsabilización del paciente en el proceso terapéutico.

Hasta ahora hemos hablado de los programas de tratamiento integral de los TP/TLP en los hospitales de día y en el caso especial de las unidades específicas de hospitalización. En esta revisión bibliográfica nos ha sido muy difícil, por no decir imposible, hallar trabajos publicados que estudiasen la eficacia del tratamiento integral a nivel ambulatorio o en hospitalización parcial. Los trabajos más recientes publicados sobre programas específicos para TLP/TP se centraban en el programa STEPPS (Systems Training for Emotinal Predictability and Problem Solving) un programa en el que se trabaja la respuesta emocional de los pacientes y la resolución de problemas en el que, para explicarlo de forma sencilla, a medida que se evoluciona en el programa se disminuye el número de sesiones hasta el alta definitiva. A modo breve resumen el programa consta de tres pasos: el primero de ellos consiste 
ORIGINALES Y REVISIONES

en explicar qué caracteriza este trastorno, las posibilidades de cambiar aspectos de éste y la responsabilidad del usuario en el tratamiento; el segundo se centra en el entrenamiento en habilidades para el manejo de las emociones y finalmente en el tercero se entrenan habilidades para el manejo de las alteraciones de conducta (13). Este programa se realiza sobre todo en hospitales de día, ya que inicialmente requiere un abordaje diario intenso. Esto, no implica que sólo se publiquen trabajos sobre este programa, pero los otros estudios publicados (la gran mayoría de los artículos encontrados) se centraban sobre todo en programas psicoterapéuticos específicamente y éste no era el objetivo de dicha revisión. También indicar, que en los estudios publicados sobre el programa STEPPS, éste solía compararse con el tratamiento habitual o únicamente con psicoterapias específicas para TLP/TP, no con otros programas, por lo que los resultados obtenidos por sí podrían ser cuestionables. Los trabajos hallados, parece que demuestran la eficacia de dicho programa cuando se compara con el tratamiento habitual ambulatorio o con una psicoterapia específica.

El más reciente, de Blum, St John y cols del 2008 (14), se trata de un ensayo clínico que compara el programa STEPPS en pacientes, que además realizan el tratamiento habitual ambulatorio, con el tratamiento ambulatorio exclusivamente hallando que la combinación de los dos tratamientos mejoraba la afectividad, la cognición, las relaciones interpersonales y la impulsividad (según la Zanarini Rating Scale for Borderline Personality Disorder (15) así como disminuía el número de visitas a urgencias, sin detectar cambios en el número de tentativas autolíticas, de autolesiones o de hospitalizaciones de los pacientes. Es decir, que el programa STEPPS más el tratamiento ambulatorio individual mejoraban en el TLP la sintomatología conductual y afectiva, lo que implicaría una mejoría del funcionamiento global del paciente. Por otro lado sería interesante determinar, en el tratamiento integral del TLP/TP, si existen algunas características del paciente que nos puedan ayudar a predecir los resultados de los diferentes tratamientos de cara obtener la máxima mejoría del paciente y optimizar recursos. Chiesa y Fonagy, con este objetivo, publicaron en 2007 (16) un ensayo clínico comparando dos programas psicosociales diferentes para los TP de clúster B, uno de los programas consistía en un tratamiento hospitalario de larga duración y el otro del programa STEPPS. Sus resultados mostraron que en los dos programas a menor edad, mayor duración del tratamiento, mejor funcionamiento global junto con falta de autolesiones predecía mejores resultados en los dos años posteriores. Cuando existían autolesiones el programa STEPPS era el que mejores resultados proporcionaba. Debemos, pero tener en cuenta que el tamaño de la muestra $(\mathrm{N}=74)$ no permitió realizar un análisis de regresión por lo que los datos obtenidos son orientativos. Grazt, Lacroce y cols publicaron en 2006 (17) un estudio prospectivo para evaluar si un tratamiento breve pero intensivo inicialmente a nivel de hospitalización parcial y posteriormente am- 
bulatorio producía mejorías en los pacientes con TLP/TP. En este estudio durante 8 semanas se realizó tratamiento intensivo siguiendo el programa STEPPS en hospital de día y posteriormente seguimiento intensivo ambulatorio con el tratamiento habitual durante un mes. Los resultados mostraron una mejoría significativa de la afectividad y del control de las emociones, una disminución de las conductas parasuicidas y de la severidad de los síntomas. Sin embargo, no mejoraron ni el funcionamiento global ni la calidad de vida. Concluyeron que era posible ver cambios en la sintomatología ya en las fases iniciales del tratamiento con terapias breves en pacientes con TLP, lo que podría ser un buen indicador de respuesta al tratamiento aumentando la efectividad y eficacia de éste. Las limitaciones de este estudio es que como método de evaluación de los resultados se realizaron autoencuestas así como el tamaño de la muestra $(\mathrm{N}=36)$ que pueden causar diferentes sesgos.

No cabe duda, que saber si un programa es más eficaz que otros o qué características del paciente determinan uno u otro es importante al implementar un programa pero no debemos olvidar que también hay que valorar si dicha eficacia se mantendrá en el tiempo. Chiesa y Fonagy (18) en 2006 publicaron un estudio de cohortes retrospectivo de los últimos seis años en el que se comparaban tres cohortes de pacientes que realizaban tratamientos diferentes, una de ellas realizaba el programa STEPPS, otra un tratamiento de larga duración hospitalario y la tercera únicamente tratamiento psiquiátrico habitual. En esos seis años de seguimiento se valoró a los 6 , 12, 24 y 72 meses la severidad del TP, el ajuste social y el funcionamiento global, las autolesiones, las conductas parasuicidas y el número de reingresos concluyendo que: el programa STEPPS lograba mejorías más significativas en la mayoría de variables y que estas mejorías se mantenían a los 6 años. El inconveniente al interpretar los resultados de este estudio es que las muestras no fueron randomizadas, que existían factores de confusión asociados a la localización geográfica de la muestra con posibilidad de diferencias entre grupos que debemos tener cuenta.

Hemos visto a lo largo de esta revisión que el programa STEPPS es uno de los más extendidos para el tratamiento integral del TLP grave. En los diferentes trabajos revisados parece que aunque en la práctica clínica es eficaz los estudios, por sus dificultades de realización en general, no han podido corroborarlo. Van Weel, Kockmann y cols (19) publicaron en 2007 un trabajo donde se explicaba la implantación del programa STEPPS en Holanda en 1998 y las causas de su rápida diseminación.

Estos autores hallaron como causas de su diseminación la manualiazición del tratamiento (que facilita su aplicación y la formación de terapeutas que junto con la brevedad de su duración (20 semanas) facilitaba el cumplimiento terapéutico. Además, por los mismos motivos, este programa era fácilmente adaptable para otro tipo de usuarios (por Ej. adolescentes). Llama, sin embargo, la atención que no se citase como causa de su amplia implantación su efectividad, por lo que cabe 
ORIGINALES Y REVISIONES

preguntarse si el mero hecho de necesitar un programa para estos pacientes hace que se escoja el menos difícil de aplicar y de más fácil "cumplimiento" sin tener en cuenta si realmente es efectivo para nuestros pacientes. También, somos conscientes que cualquier tipo de intervención terapéutica en estos pacientes es efectiva y simplemente el conseguir una adherencia durante un tiempo mínimo a un tratamiento con estos pacientes nos permite casi asegurar alguna mejoría en algún ámbito.

A lo largo de esta revisión hemos focalizado el tratamiento integral del TLP en hospitales de día, pero en los últimos años se están implantando en los CSM programas especializados para TLP con buenos resultados. Una revisión de los estudios sobre estos últimos (20) concluye que los datos sugieren que 10 horas semanales de tratamiento especializado son suficientes en los trastornos graves de personalidad, pero finaliza indicando la falta de estudios que valoren si la efectividad de dichos tratamientos a nivel de CSM es igual o superior a la de los hospitales de día.

Como podemos suponer en estos pacientes el núcleo familiar y social es básico ya que son los que, después del paciente, más se ven afectados por la inestabilidad que caracterizada a los pacientes con TLP. Preguntándose en muchas ocasiones si ellos son la causa de lo que le sucede, qué pueden hacer para ayudarlo y porqué tras acudir a urgencias de nuestros servicios suele indicárseles la inconveniencia de un ingreso hospitalario y se les remite de nuevo a su casa con el paciente sin haberles proporcionado, normalmente, el soporte que ellos demandan (que por regla general suele ser el ingreso). Si embargo, aunque en la mayoría de tratamientos se realizan intervenciones familiares, sería interesante valorar el papel de la utilidad de la psicoeducación en estas familias de cara a comprender qué le sucede al paciente, cómo ayudarlo y qué pueden hacer en situaciones de crisis para evitar acabar en urgencias con el paciente (normalmente tras realizar algún gesto autolítico). Hoffman y Fruzzetti (21) publicaron en 2007 una revisión de trabajos sobre psicoeducación en familiares de pacientes con TLP y encontraron que existían muy pocos programas para familias de pacientes con TLP/TP a pesar de la importancia de éstas en el tratamiento, concluyendo que aunque los escasos estudios hallados al respecto sugerían la utilidad de la psicoeducación en familias de pacientes con TLP ésta era muy infrecuente en los programas.

\section{Conclusiones}

La impresión clínica general es que en los últimos años se observa un incremento de pacientes diagnosticados de TLP. Este incremento, ¿es real o es que 
actualmente se diagnostican más por mayor facilidad para acudir a los servicios de salud mental que hace unos años? Si este incremento es real, ¿cuál es la causa, social, por criterios diagnósticos, etc...?.

Actualmente, las diferentes redes de salud mental ya disponen de programas concretos para los pacienten con TLP, éstos ¿son realmente necesarios y eficaces?, ¿son suficientes para la demanda?, ¿se deben realizar pensar en psicoeducativos para el núcleo de convivencia?

La mayoría de trabajos publicados en relación a programas específicos para los TLP se centran en el programa STEPPS y parece que la mayoría de Hospitales de Día españoles siguen pautas básicas parecidas. Esto, podría sugerir que tal quizás éste sea el tratamiento más eficaz. Además, parece que excepto en los casos de TP más graves, el tratamiento debe realizarse a nivel comunitario más que hospitalario. Por otro lado, las unidades de hospitalización específicas para estos trastornos están surgiendo como alternativa terapéutica generando controversia entre los profesionales.

Por último, existe una falta de estudios que avalen la eficacia del tratamiento integral del TLP (no así del tratamiento psicoterapéutico), a pesar que sí hay una clara evidencia clínica de su eficacia. Entre las múltiples causas de esta escasez de estudios destacan las relacionadas con las dificultades para diseñarlos, seguramente en relación a la cantidad de diferentes variables a tener en cuenta, la alta tasa de abandonos (por las propias características de estos pacientes) y las dificultades para comparar las estrategias terapéuticas con un placebo entre otras muchas.

DIRECCIONES DE INTERÉS:

(A) Barnet, Enfield \& Haringey Mental Health nhS Trust. Dr A.W. Bateman Consultant Psychiatrist in Psychotherapy Halliwick Specialist Service. St Ann's Hospital St Ann's Road, TotTenham. London N15 3TH. TelePhone: (020) 84426528

(B) Programa PILOTO de atención InTEgrada TLP y TP EN LOS CENTROS asistenciales DE Torribera. Director: Dr. Fernando Lana Moliner (Hospital de Día). Cap del Departament de Psiquiatria i Salut Mental Centres Assistencials Torribera, c/ Prat de la Riba, 17108921 Santa Coloma de Gramenet. Barcelona. Teléfono: (93 46289 00)

(C) UTP-FundipP (Unidad Trastornos Personalidad Cantabria): Plaza del Cuadrado 3, 3ํ B y C. CP 39004 SANTANDER. TelÉF: 902390001 / FAX: 942223894. E-Mail: GESTION@ @undipp. ORG

(D) Centro Asistencial San Juan de Dios (Málaga). Dpto. de Admisiones: Dr Conrado Muñoz y Dr. Juan Luís Morales. TeléF: 952250550

(E) Programa integrado de Trastornos de Personalidad del CSM Arganda. Director: Dr. Luis Javier Irastorza Eguskiza. Centro de Salud Mental de Arganda. Arganda del Rey, MaDRID. TEL: 18713081-918700417 - FAX: 918703937 
(F) Parc Hospitalari Martí I Julià. Hospital de Día (Dr Enrique García Muñoz): C/ Dr Castany s/n, CP 17190, Salt (Girona) Teléf: 972182576

(G) Unidad de Trastornos de la Personalidad del Hospital Provincial de Zaragoza. Director: Dr. Vicente Rubio Larrosa. Prof de Psiquiatria. Universidad de Zaragoza. Hospital Provincial, "Nuestra Señora de Gracia", Servicio de Psiquiatria. C/Ramon y Cajal, 60, 50004 Zaragoza. Tel 976 440022

\section{BIBLIOGRAFÍA:}

(1) Gunderson, John G. Trastorno Límite de Personalidad: Guía Clínica. Ed. RBA, 2002. Barcelona

(2) Oldham, J. M. Tratado de los Trastornos de Personalidad. Barcelona: Ed Masson, 2007; p. 59-90.

(3) Szerman Bolotner, Néstor; Pérez Díaz, Ma Dolores; Ruíz Sancho, Ana. Trastorno Borderline de la Personalidad. Barcelona: Ed Entheos, 2004.

(4) Lana Moliner F, González Torres MA, Mirapeix C. Niveles asistenciales y requisitos organizativos en el tratamiento de los trastornos de personalidad. Psiquiatria.com [revista electrónica] 2006; 10 (1 Suppl): Disponible en http://www.psiquiatria.com/articulos/tr_personalidad_y_habitos/26268/

(5) American Psychiatric Association. Guía Clínica para el tratamiento de los Trastornos Límite de Personalidad. Barcelona: Ed. Ars Medica,2002.

(6) BeCK A; ET AL. Principios Generales de la Terapia Cognitiva de los Trastornos de Personalidad. Barcelona. Ed Paidós, 1995.

(7) Linehan, Marsha M. Manual de tratamiento de los Trastornos de Personalidad Límite. Barcelona: Ed Paidós, 2003.

(8) Rodríguez Cahill C, Murias Fernández E. Situación actual de los tratamientos en el trastorno límite de personalidad. Psiquiatria.com [revista electrónica] 2006; 10 (1 Suppl): Disponible en http://www.psiquiatria.com/articulos/tr_personalidad_y_habitos/26233/

(9) Boyra A, Maruottolo C, Mascaró A, Guimón J. Resultados de la psicoterapia grupal breve dinámica en pacientes borderline. ASMR Revista Internacional On-Line[revista electrónica] 2007; Jul: $6(2)$

(10) Grupo TlP de FEAFES Valladolid El Puente. Trastorno Límite de Personalidad: Unidades Específicas TLP.

(11) Clarkin JF, Lvy KN, Lenzenweger MF, Kernberg OF. Evaluating three treatments for borderline personality disorder: a multiwave study. Am J Psychiatry. 2007 Jun; 164 (6 Suppl): 922-8.

(12) PARIS J. Intermitent psychotherapy: an alternative to continuos long-term treatment for patients with personality disorders. J Psychiatr Pract. 2007 May; 13 (3 Suppl): 153-8.

(13) The STEPPS Group Treatment Program for Borderline Personality Disorder. www.uihealthcare.com/topics/medicaldepartments/psychiatry/stepps/index.html

(14) Blum N; St John D; Pfohl B; Stuart S; McCormick B; Allen J; Arndt S; Black DW. Systems Training for Emotional Predictability and Problem Solving (STEPPS) for Outpatients With Borderline Personality Disorder: A Randomized Controlled Trial and 1-Year Follow Up. Am J Psychiatry. 2008 Apr; 165 (4): 468-478 
(15) Zanarini MC, Vujanovic AA, Parachini EA, Boulanger JL, Frankenburg FR, Henner J. Zanarini Rating Scale for Borderline Personality Disorder (ZAN-BPD): a continous mesure of DSM-IV borderline psychopatology. J. Pers. Disord. 2003 Jun; 17 (3): 233-42

(16) ChiEsa M, Fonagy P. Prediction of medium-term outcome in cluster B personality disorder following residential and outpatient psychosocial treatment. Psychother Psychosom. 2007; 76 (6): 347-53.

(17) Grazt KL, Lacroce DM, Gunderson JG. Mesuring changes in symptoms relevant to borderline personality disorder following short-term treatment across partial hospital and intensive outpatient levels of care. J Psychiatr Pract, 2006 May; 12(3):153-9.

(18) Chiesa M, Fonagy P, Holmes J. Six-year follow-up of three programs to personality disorder. J Personal Disorder, 2006 Oct; 20 (5): 493-509.

(19) Van Wel B, Kockmann I, Blum N, Pfohl B, Black DW, Heesterman W. STEPPS group treatment for borderline personality disorder in The Nederlands. Ann Clin Psychiatry, 2006 Jan-Mar; 18 (1): $63-7$

(20) Karterud S, Wilberg T. From general hospital treatment to specialized treatment programs. Int Rev Psychiatry. 2007 Feb; 19 (1): 39-49.

(21) HofFman PD; FruZzeTtI AE. Advances in interventions for families with a relative with a personality disorder diagnosis. Curr Psychiatry Rep, 2007 Feb; Vol. 9 (1), pp. 68-73 